Electronic Journal of Statistics

Vol. 15 (2021) 3948-3950

ISSN: $1935-7524$

https://doi.org/10.1214/21-EJS1886

\title{
Corrigendum to "Quantification of the weight of fingerprint evidence using a ROC-based Approximate Bayesian Computation algorithm for model selection"*
}

\author{
Jessie Hendricks and Cedric Neumann and Christopher P. Saunders \\ Department of Mathematics and Statistics, \\ South Dakota State University, \\ Brookings, SD, USA \\ e-mail: \\ jessiehhendricks@gmail.com; cedric.neumann@me.com; christopher.saunders@sdstate.edu
}

\begin{abstract}
In this correction, we provide an acknowledgment for the source of several images in the paper: J. Hendricks, C. Neumann, C.P. Saunders (2021) "Quantification of the weight of fingerprint evidence using a ROCbased Approximate Bayesian Computation algorithm for model selection", Electronic Journal of Statistics (15)1, pp. 1228-1262.
\end{abstract}

Keywords and phrases: Approximate Bayesian Computation, Bayes factor, correction, Forensic science, fingerprint, weight of evidence, receiver operating characteristic.

Received July 2021.

In this correction, we reproduce Figures 1, 6, and 7 from J. Hendricks, C. Neumann, C.P. Saunders (2021) "Quantification of the weight of fingerprint evidence using a ROC-based Approximate Bayesian Computation algorithm for model selection", Electronic Journal of Statistics (15)1, pp. 1228-1262, and include a specific acknowledgment statement for the source of these images.

\section{Acknowledgments}

We would like to thank the Latent Print Unit of the Denver Police Department, Crime Laboratory and specifically Latent Print examiners Courtney Mohr and Elizabeth Ennenga for providing us with annotation data and images partly used in this paper. These data were collected as part of Award 2015-DN-BX-K027 from the National Institute of Justice.

\footnotetext{
${ }^{*}$ Corrected article: 10.1214/21-EJS1813.
} 

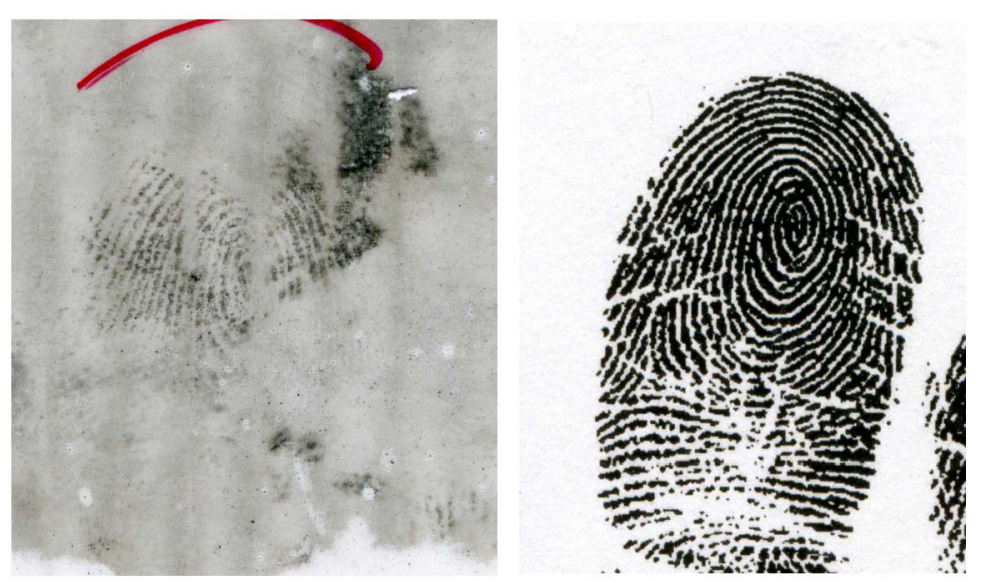

FIG 1. Reproduction of Figure 1 from Hendricks, Neumann and Saunders (2021). Left panel: fingermark. Right panel: fingerprint taken under controlled conditions. Ridges appear darker than background. Both impressions were made by the same finger. Their comparison shows that both ridge flows are affected by different distortion and degradation effects. Fingermark and control print images were provided by the Latent Print Unit of the Denver Police Department, Crime Laboratory.
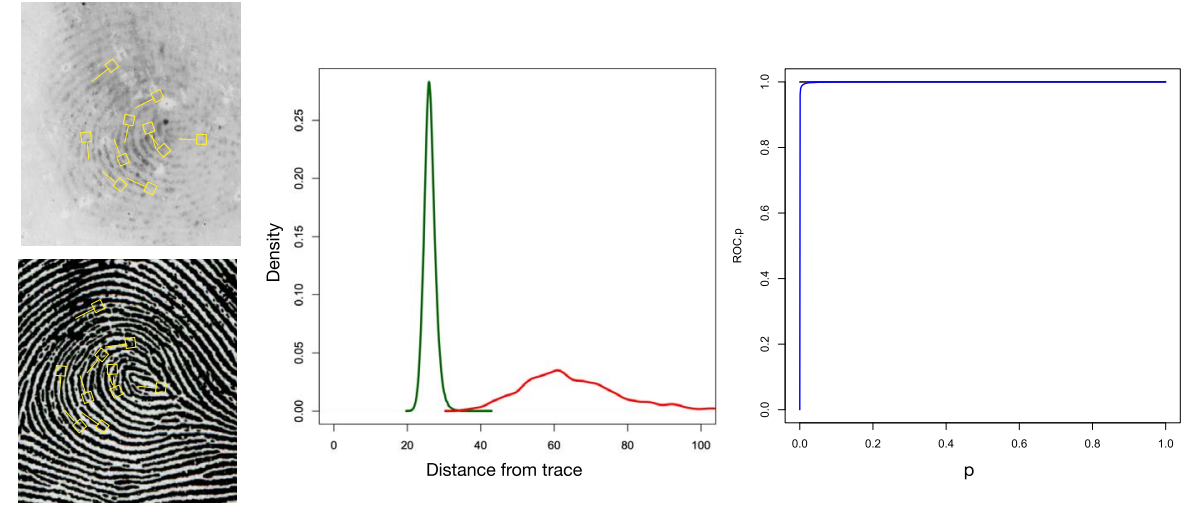

FIG 2. Reproduction of Figure 6 from Hendricks, Neumann and Saunders (2021). Left panel: Fingermark (top) and control print (bottom), both originating from the same source. Corresponding features between the two impressions have been annotated in yellow. Middle panel: Kernel density estimates of the densities of the scores generated under $\mathrm{H}_{1}$ (green) and $\mathrm{H}_{2}$ (red). Note that the distribution of scores generated under $H_{1}$ is situated much closer to 0 , but neither of the curves cover 0. Right panel: Empirical ROC curve (black) which is nearly perfectly overlapped by the parametric ROC curve (blue) generated from the distributions of scores in the middle panel. Note the steep slope of the curve near $p=0$. Fingermark and control print images and annotation data were provided by the Latent Print Unit of the Denver Police Department, Crime Laboratory. 

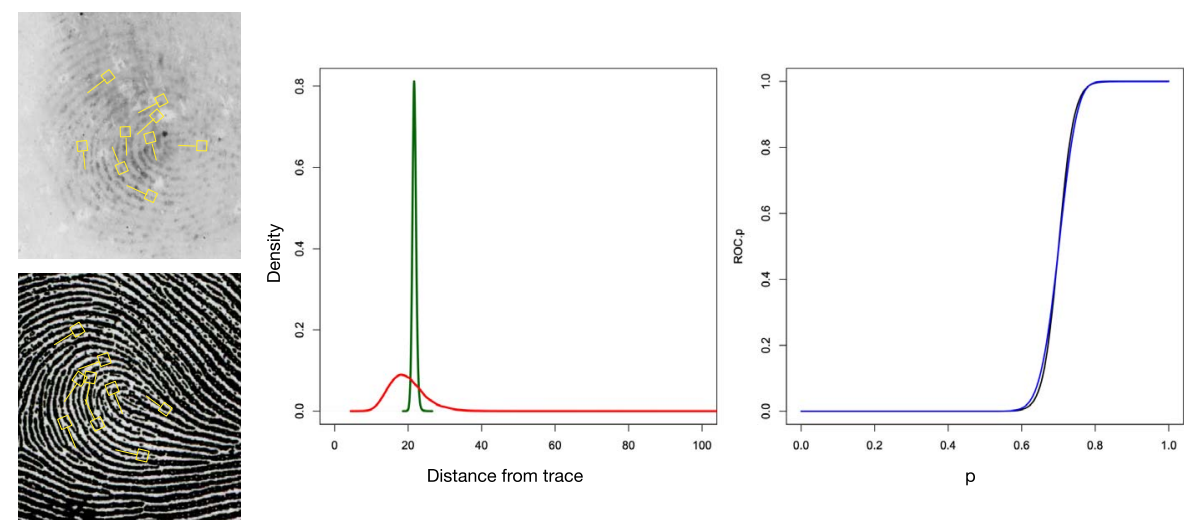

Fig 3. Reproduction of Figure 7 from Hendricks, Neumann and Saunders (2021). Left panel: Fingermark (top) and control print (bottom) originating from the different sources. Potentially corresponding features between the two impressions have been annotated in yellow. Middle panel: Kernel density estimates of the densities of the scores generated under $H_{1}$ (green) and $\mathrm{H}_{2}$ (red). Note that the algorithm has detected many more random impressions that are more similar to the fingermark than the impression from the suspect. Right panel: Empirical ROC curve (black) and parametric ROC curve (blue) generated from the distributions of scores in the middle panel. Note the flat slope of the curve near $p=0$ indicating that the algorithm supports $\mathrm{H}_{2}$. Fingermark and control print images and annotation data were provided by the Latent Print Unit of the Denver Police Department, Crime Laboratory.

\section{References}

Hendricks, J. H., Neumann, C. and Saunders, C. P. (2021). Quantification of the weight of fingerprint evidence using a ROC-based Approximate Bayesian Computation algorithm for model selection. Electronic Journal of Statistics 15 1228-1262. MR4255299 